\title{
AQUiD: Automated Quality Assessment Using Digital Image Processing
}

\author{
${ }^{1}$ Nirmal Dorothy, ${ }^{2}$ Abid Rahman, ${ }^{3}$ Ajmal Ashik, ${ }^{4}$ Dr. Rekha Lakshmanan \\ ${ }^{1,2,3}$ UG Scholar, ${ }^{4}$ Professor, KMEA Engineering College, Aluva, India. \\ 1'nirmal.dorothy@gmail.com, ${ }^{2}$ aabidrahman990@gmail.com, ${ }^{3}$ munnaashik786@gmail.com, \\ ${ }^{4}$ rekhavibin@gmail.com
}

\begin{abstract}
Products in the market are expected to satisfy the consumer's quality requirements. Agriculture being one of the main occupation of the people of India, the raw products must be sorted to determine whether they fit the quality description so that high quality products are obtained as the end result. The proposed method is designed to ensure the availability of good quality coconut oil in the market by assessing the quality of each individual sample going into the production line. $70 \%$ of moisture content present naturally in copra(dried coconut kernel) is dried to almost $7 \%$ for coconut oil production. To prevent the growth of bacteria and fungus on the surface of the copra, sulphur is added as a preservative. Allergenic reactions and lung performance restrictions can be caused due to the presence of sulphur in copra. The presence of moisture may also adversely affect oil quality. The texture features such as wrinkles, moulds, fungi growth on the surface also deplete the oil quality. The features of different kinds of copra are analysed and is used train the machine. The machine learning methodology is adopted for the classification of copra as usable and unusable.
\end{abstract}

Keywords - Agriculture, classification, copra, image processing technique, machine learning, sulphur

\section{INTRODUCTION}

In the economic development of India, agriculture plays a major role. The demand for coconut and its related products are always increasing in the market. The quality of coconut is degraded by contamination by many factors leading to its spoilage. There is enough possibility for fungal and bacterial infections over the products due to high humidity in the atmosphere. If not dried properly, the level of moisture content present may deteriorate the quality of copra.

The farmers use the process of sulphur fumigation over the coconuts in order to avoid infections and to keep the coconuts in a good condition, ready to be sold out to the merchants. Sulphur patch is formed India is the third largest coconut producer in the world with a production of over 119 million tones and consumption of 385 kilo tones of coconut oil.

Today Kerala produces roughly $45 \%$ of India's coconuts, with some $92 \%$ of total production lying in the southern Indian states and Kerala's neighbors.The proposed methodology aims to provide support to oil manufacturing units to assess the quality of copra samples taken for oil production. The main parameters against which the quality is assessed are- moisture content of copra, presence and percentage of sulphur present in copra and discoloration on the surface. Assessment of these factors can help in determining whether each sample going into production of oil fits the quality description and whether they are to be discarded or not.

Digital image processing technology can be effectively employed to perform the segregation. The method involves capturing of images so that the features of each independent sample can be studied. This is followed by the application of enhancement techniques in order to highlight and extract the distinguishing features. The enhanced image is then subjected to segmentation process where the region of interest is segmented from the background. Then the features of every individual sample of copra are analyzed which will be used for training purposes. A classification algorithm can be used to differentiate normal copra from the deteriorated copra .

\section{RELATED WORKS}

Sagayaraj, et.al [1] established methodology for detecting presence and percentage of sulphur in copra[1].The sulphur added copra can be differentiated from normal copra by studying the RGB colour features. The decreasing shape features was also studied to identify the sulphur present in the copra . K-means clustering technique and the KNearest Neighbour classification were employed.

Sagayaraj et. al [2] discussed the different textural, shape and colour features of the copra that can be measured to 
classify copra. These factors were applied to the classifiers which underwent analysis to assign the fruits to the corresponding quality categories using statistical and syntactical classifiers.

Mahendran et.al [3] focused on the recent development and application of image analysis and computer vision system in quality evaluation of products in the field of agriculture. The author also discussed the hardware and software requirements and developments in recent time, giving emphasis on monochrome imaging, colour imaging and multispectral imaging for modern grading and sorting systems.

Vision based features in moisture content measurement were studied by Sagayaraj et. al [4] where primary focus for studying the characteristics of fruits were based on its moisture content. The main features that undergo change during drying are the shape, color and texture feature. It was observed that the features decreases smoothly with drying time. The moisture content were predicted by employing the ANN models. The integration of neural network proved to be an efficient and proper tool to predict the moisture content.

The quality evaluation of fruits and vegetables using computer vision was reviewed by Bhargava et. al [5] and observed that in agriculture science, the quality can be increased by automated processes. If the sorting process is done manually the process of sorting and grading will become inconsistent, time consuming, variable, subjective, onerous, expensive and easily influenced by surrounding. The author also carried out critical comparison of different algorithm proposed by different researchers for quality inspection of fruits and vegetables.

Patel KK et. al [6] discussed how machine vision can act as a tool for quality inspection of food and agriculture and observed that the quality inspection of food and agricultural produce are difficult and labor intensive at the same time. With increased expectation for food products of high quality and safety standards, the need for accurate, fast and objective quality determination of these characteristics in food products continues to grow. The paper shows how machine vision provides one alternative for an automated, non-destructive and cost-effective technique to accomplish these requirements. this inspection approach based on image analysis and processing has found a variety of different applications in the food industry.

Patel KK et. al[7] reviewed image processing tools and techniques used in computer vision for quality assessment of food products. This paper reviews developments of image processing tools and techniques for food quality evaluations, which include principles of image processing; imaging systems; and low-, mid- and high-level image processing. Low-level image processing involves noise removal and contrast enhancing, mid-level image processing requires segmentation based on thresholding, gradient, region, and classification methods are also included. The authors discussed the significant elements and important aspects of image processing techniques used in computer vision systems and emphasizes the considerable research of image processing techniques for the inspection and grading of food products.

A fruit quality management system based on image processing was developed by Ms.Rupali et. al[8]. The author focuses on two choices for grading i.e, by color and size. In first case sorting circular shaped fruits according color and grading is done according to size. The proposed automated classification and grading system is designed to combine three processes such as feature extraction, sorting according to color and grading according to size. The author designed the entire system over MATLAB software to inspect the color and size of the fruit. System can sort and grade the fruits according to different attributes such as color and size.

\section{MeThodology}

The proposed methodology aims to provide support to oil manufacturing units to assess the quality of copra samples taken for oil production. The main parameters against which the quality is assessed are- moisture content of copra, presence of sulphur present in copra and discoloration on the surface.

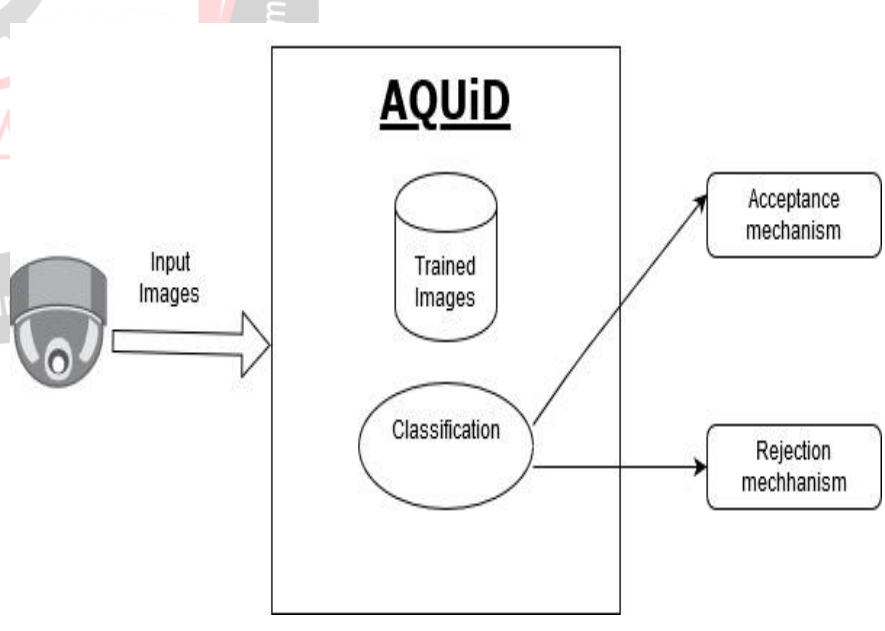

Fig 1: Use case diagram

Figure 1 shows the use case diagram of the proposed system. The proposed method is designed in such a way that an input image can be tested to determine whether it meets the required quality standards and thereafter engaging a suitable acceptance or rejection hardware mechanism based on the classification output. The four major modules of the proposed method includes:
I) Image aquisition
II) Preprocessing and segmentation
III) Feature extraction
IV) Classification 
Image Aquisition



Fig 2: Capturing of image.

In the proposed method, collecting images of copra belonging to various categories is the first step involved. A real database consisting of different kinds of copra such as mouldy copra, copra with sulphur content, copra with moisture content, copra with wrinkles, and good copra samples is maintained to be used for training purposes. The images are captured so that the characteristics of different samples can be studied.

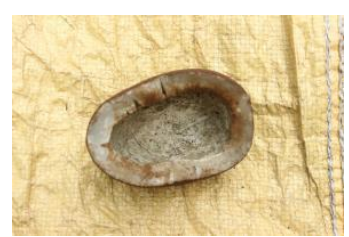

(a)

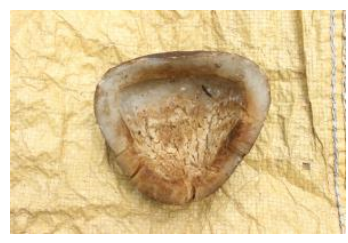

(c )

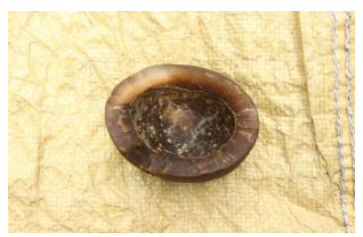

(e )

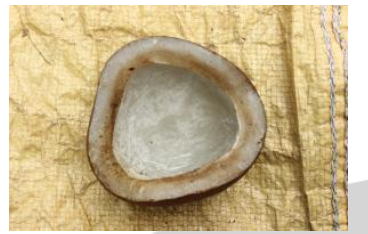

(b)

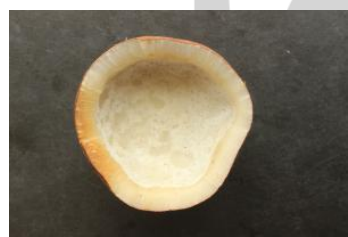

(d)

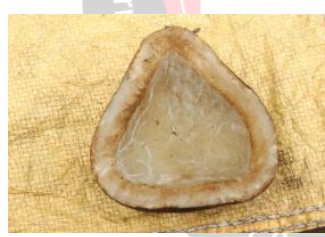

(f)
Fig 3: Images of different categories of copra (a)Mouldy copra, (b)Moist copra, (c)Wrinkled Copra, (d)Copra with sulphur content, (e)Unusable copra, (f)Usable milling copra.

Hundreds of such images were collected and used for training purposes. The images were captured using Canon EOS 600D 18MP Digital SLR professional camera.

Pre-processing and Segmentation

To extract basic information from a digital image, basic image enhancement techniques are adopted. Pre- processing of copra images improves the visibility of edges and reduction of noise in the captured image. This is done in order to highlight the distinguishing features of different categories of copra. Atmospheric haze reduction is performed on the obtained image as a part of enhancement process.To obtain the region of interest from the original image, the small holes and gaps in the image were filled using morphological operation and structural element shaped as a disk of specific radius was used in the opening function to perform the task. Red, green and blue planes were separately extracted from the image. This is particularly useful for images where background removal becomes a difficult task due to the similarity in colour information of background and foreground pixels.

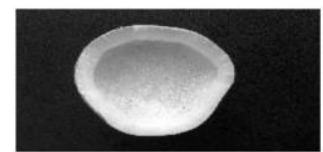

(a)

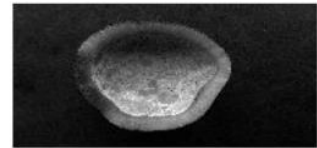

(c)

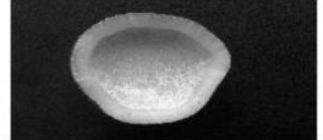

(b)

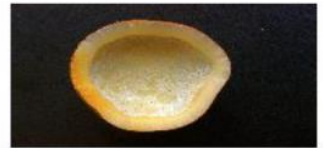

(d)
Fig 4: RGB planes of input image. (a) Red plane, (b) Green plane, (c) Blue plane, (d) Original copra image

Binarisation of the images are done and sum of the RGB planes are obtained. The level of threshold is determined by using graythresh function. The background and foreground pixels of the image should be ideally represented by intensity values 0 and 1 respectively. In order to achieve this complementation of the image obtained as the sum of the three planes is taken.

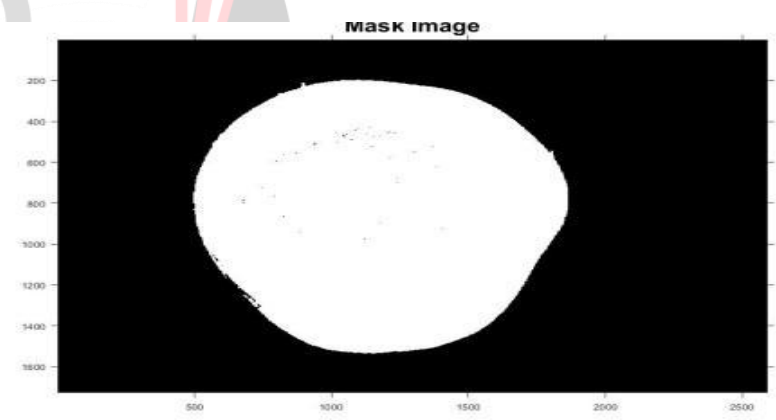

Fig 5: Binarised image of the sum of planes

This mask image is superimposed over the original RGB image thereby highlighting the region of interest.

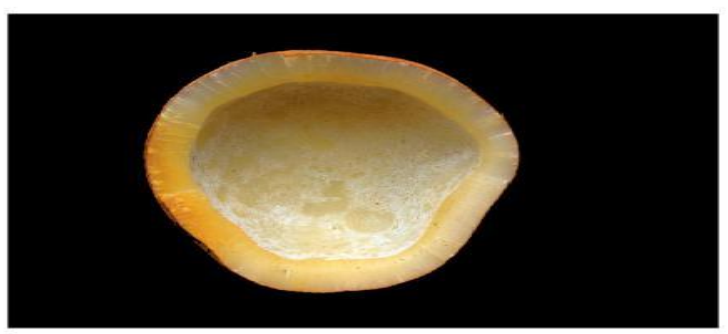

Fig 6: Segmented region of interest

Feature Extraction

From the image data, the features at various levels of complexity are extracted. From the segmented ROI of the 
image specific set of interest points from the original image are isolated. On inferring the values of different functions applied on this specific area unique characteristics of individual samples of copra can be analysed. To differentiate the sulphur added copra from normal copra the RGB colour features are extracted. During drying process the shape and statistical features are analysed to identify the sulphur content in copra.

Some of the features of the copra that can be analyzed to determine whether it is fit for oil production are mean of the intensity of the pixels, standard deviation of the intensity values of the pixel, RGB values of the pixels, length of major axis and minor axis, Correlation Coefficient etc.

The different feature values are stored as a csv file to provide as an input for training and classification. Among the observed feature values only those features whose values that differs considerably for different classes are included in the dataset for training.



Fig 7: Csv file of extracted feature values

To differentiate between unusable copra and normal usable copra the specific set of features includes the separate mean values of red, green and blue planes and the mean of $\mathrm{V}$ pixels of the corresponding HSV image. Likewise, for classification into into different classes different feature value sets are considered.

\section{Classification}

The primary classification is to differentiate extreme bad copra and the usable ones followed by classification of normal copra from sulphur added copra. Then other copra with high moisture content, presence of moulds on the surface, wrinkled copra are classified. The machine is trained using values obtained during the previous stage of processing. To distinguish copra from good and not usable.

The following figure shows a sample scatter plot depicting the relationship between variables representing different feature values of good and bad copra. The position of each dot on the horizontal and vertical axis shows values for an individual data point.

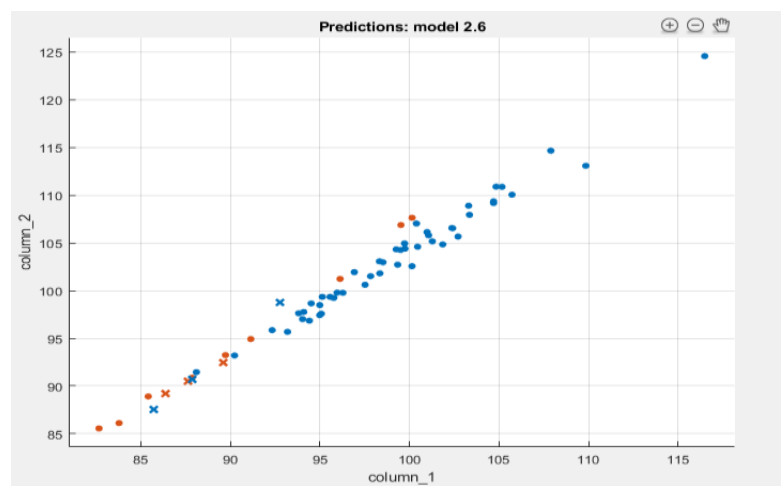

Fig 8. Sample scatter plot of classification

The different coloured dots show data points corresponding to two different classes, say, good and bad copra. Ideally, for classification the different coloured dots are not expected to occur at any region simultaneously. In association with the given figure, if the red dots are completely concentrated on the lower area of the graph and the blue dots are distinguish-ably apart from the red ones, then higher accuracy can be achieved.

\section{RESULTS AND DISCUSSION}

By analysing the different feature values for different classes of copra it is highly visible that some feature values differ considerably aiding the process of classification. The adjoining two tables shows the sample varying values of different samples.

\begin{tabular}{|l|l|l|l|l|}
\hline $\begin{array}{l}\text { Mean of } \\
\text { pixels }\end{array}$ & $\begin{array}{l}\text { Mean of red } \\
\text { pixels }\end{array}$ & $\begin{array}{l}\text { Mean of } \\
\text { green pixels }\end{array}$ & $\begin{array}{l}\text { Mean of } \\
\text { blue pixels }\end{array}$ & $\begin{array}{l}\text { Mean of V } \\
\text { pixels of } \\
\text { HSV image }\end{array}$ \\
\hline 83.7898 & 86.1088 & 83.6250 & 83.6250 & 86.1263 \\
\hline 82.6753 & 85.5454 & 82.5137 & 82.5137 & 85.5612 \\
\hline 85.7576 & 87.5587 & 85.9359 & 83.7781 & 87.5782 \\
\hline 85.4235 & 88.9019 & 85.2556 & 82.1130 & 88.9213 \\
\hline
\end{tabular}

Table 1. Feature values of unusable copra samples.

This data set is given as input data set for training in the Classification Learner App in MATLAB. For classifying extremely bad and good copra Linear Regression showed a high accuracy of $90.4 \%$ and for classifying the sulphur added copra from normal copra SVM showed 100\% accuracy.

\begin{tabular}{|l|l|l|l|l|}
\hline $\begin{array}{l}\text { Mean of } \\
\text { pixels }\end{array}$ & $\begin{array}{l}\text { Mean of red } \\
\text { pixels }\end{array}$ & $\begin{array}{l}\text { Mean of } \\
\text { green pixels }\end{array}$ & $\begin{array}{l}\text { Mean of } \\
\text { blue pixels }\end{array}$ & $\begin{array}{l}\text { Mean of V } \\
\text { pixels of } \\
\text { HSV image }\end{array}$ \\
\hline 99.2661 & 104.3219 & 99.9118 & 93.5648 & 104.3455 \\
\hline 95.7767 & 99.2365 & 96.2260 & 91.8675 & 99.2597 \\
\hline 98.5183 & 102.9703 & 98.7447 & 93.8400 & 102.9902 \\
\hline 101.2773 & 105.1624 & 101.8650 & 96.8045 & 105.1931 \\
\hline
\end{tabular}

Table 2. Feature values of usable copra samples 
The adjoining figure shows a sample confusion matrix in which the total number of observations in each cell is displayed. The rows of the matrix correspond to the true classes while the column corresponds to the predicted classes. The diagonal and off- diagonal cells correspond to correctly and incorrectly classified observations, respectively.

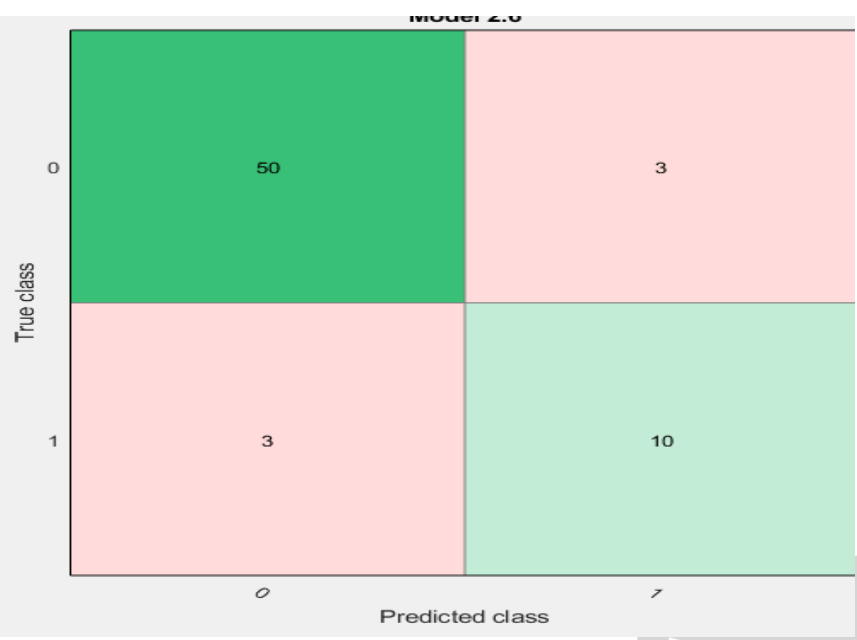

Fig 9: Confusion matrix of a sample input data set.

The trained model can then be exported to predict whether the feature values of an input image belongs to usable or unusable class.

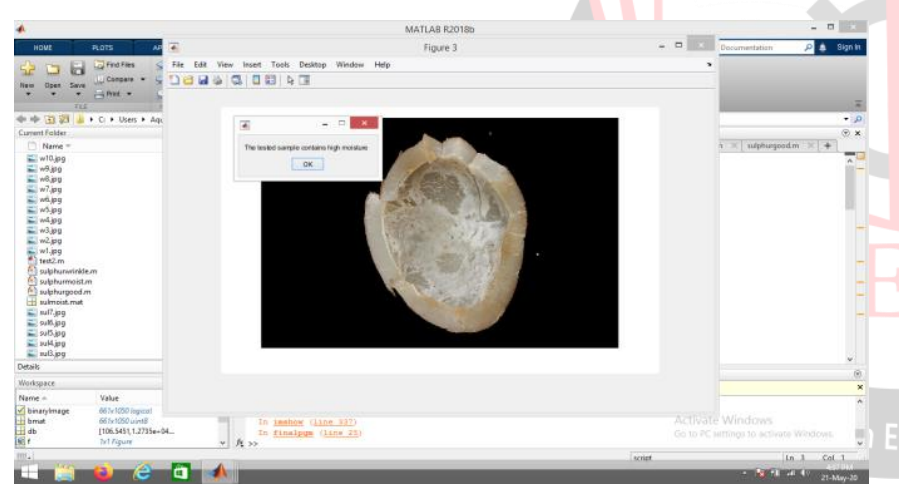

(a)

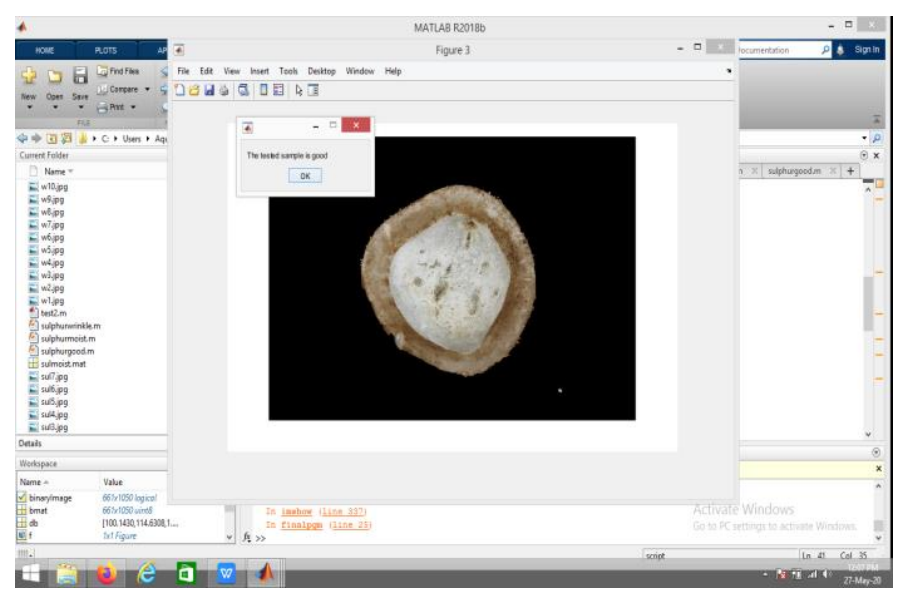

(b)

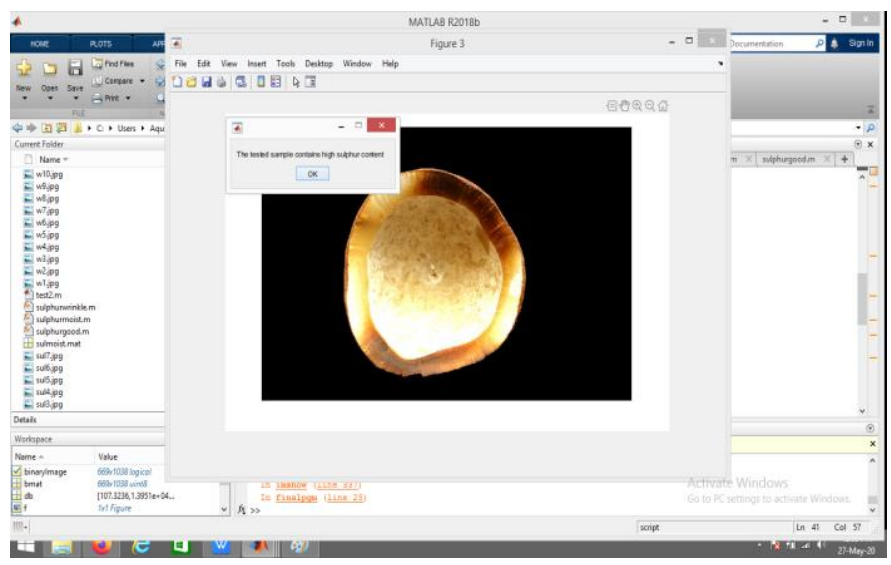

(c)

Fig 10: Sample output screen shots

The system provides a text box along with the original image representing the class to which the image sample corresponds.

\section{CONCLUSION}

The ability to generate precise descriptive pictorial information have led to the widespread use of digital image processing technology. Quality control in combination with the increasing automation in all fields of production has led to increased demand for automatic and objective evaluation of different products.A computer vision system is found to be more efficient with automatic handling mechanism so that it could perform inspections objectively and reduce tedious human involvement.

The basic objective of the proposed methodology is to develop an innovative and effective method for automatic assessment of quality of copra, the dried kernel of coconut. Early detection of defective copra provides support to oil manufacturing units to assess the quality of copra samples taken for oil production. The main parameters against which the quality is assessed are- moisture content of copra, presence and percentage of sulphur present in copra, mouldy substances on the surface, wrinkles and discolouration on the surface of copra. The proposed methodology has efficiently achieved the objective. The method, designed over Matlab can produce results within seconds of processing and a high accuracy of $94.4 \%$ is obtained for sorting process.

To measure quality consistently machine image technology provides a rapid, alternative means. It also has the potential to eliminate humans effort involving visually intensive work. Since the manual labour involved in the process is reduced considerably and is also expected to have a direct impact in reducing the cost of coconut oil in the market.

\section{ACKNOWLEDGMENT}

We would like to thank KMEA Engineering College for providing research facilities and to Prof. Dr.Rekha 
Lakshmanan (Department of Computer Science and Engineering) for immense support, encouragement and guidance.

\section{REFERENCES}

[1] A Stephen Sagayaraj, G Ramya \& N.Dhanaraj, "Analysis of Sulphur Content in Copra" (November 2018), ICTACT Journal on Image and Video Processing, Volume:09, Issue: 02

[2] A Stephen Sagayaraj, Dr.T.Kalavathi Devi , "Characteristic Recognition of copra using Image processing” (November 2015 ), International Journal of Engineering Science Invention Research \& Development; Vol. II Issue V

[3] Mahendran R, Jayashree GC, Alagusundaram K, "Application of Computer Vision Technique on Sorting and Grading of Fruits and Vegetables", Journal of Food Processing Technology, DOI: 10.4172/2157-7110.S1-001

[4] A. Stephensagayara, R. Pradeepa, P. Suganthi, S. Vinothini, T. Vishvapriya, "Vision based Features in Moisture Content Measurement-A Survey" (February 2019), International Journal in Advanced Research in Electrical, Electronics and Instrumentation Engineering, Vol. 8, Issue 02

[5] Anuja Bhargava, Atul Bansal, "Fruits and vegetables quality evaluation using computer vision: A review" (2018), Journal of King-Saud University- Computer and Information Sciences

[6] Patel KK, Khan MA, Kar A, Kumar Y, Bal LM and Sharma DK, "Image Processing Tools and Techniques Used in Computer Vision for Quality Assessment of Food Products: A Review", International Journal of Food Quality and Safety | Year-2015 | Volume 1| Pages 01-16

[7] Patel KK, Kar A, Jha SN, Khan MA, "Machine vision system: a tool for quality inspection of food and agricultural products"(2012), Journal of Food Science Technology, DOI: $10.1007 /$ s13197-011-0321-4

[8] Rupali S.Jadhav, S.S.Patil, "A Fruit Quality Management System Based On Image Processing" (NovDec 2013), IOSR Journal of Electronics and Communication Engineering, Volume 8, Issue 6. 